\title{
Quantification of Genotoxic Impurity 2-Butyl p-toluene- sulfonate at ppm Level by LC-MS/MS in Naproxen Drug Substance
}

\author{
M. B. V. NARAYANA ${ }^{1 *}$, K. B.CHANDRASEKHAR ${ }^{2}$ and $\quad$ B. M. RAO ${ }^{3}$
}

\author{
${ }^{1}$ Analytical Research, Matrix Laboratories Limited, Hyderabad-500 072, India \\ ${ }^{2}$ Department of Chemistry, Jawaharlal Nehru Technological University College of \\ Engineering, Anantapur-515002, India \\ ${ }^{3}$ Analytical Research, Johnson and Johnson Ltd., Mumbai-400080, India \\ balajimachireddy@gmail.com
}

Received 14 December 2013 / Accepted 2 January 2014

\begin{abstract}
The objective of the present research work is to develop a suitable LC-MS/MS method for the quantitative determination of genotoxic alkylating impurity 2-butyl $p$-toluenesulfonate present in naproxen drug substance at ppm level. The LC-MS/MS method was developed on Xterra MS C18 50 $\mathrm{mm}$ column using the mobile phase consists a mixture of $10 \mathrm{mM}$ ammonium acetate and acetonirile using a isocratic composition of 20:80 v/v. Ion source is electrospray ionization (ESI) positive mode, source temperature is $300^{\circ} \mathrm{C}$, gas flow is $10 \mathrm{~L} / \mathrm{min}$, Nebuliser pressure is $50 \mathrm{psi}$, capillary voltage is $4000 \mathrm{~V}$. Under these LC and MS conditions 2-butyl $p$-toluenesulfonate was quantified by selected ion mode (SIM) of 173. The limit of detection and the limit of quantitation for the impurity were established. This method has been tested in a number of naproxen samples and used successfully for quantification of the genotoxic impurity at ppm level. Validation of the developed LC-MS/MS method was carried out as per ICH requirements and the data shows that the proposed method is specific, linear, accurate, precise and robust. The developed LC-MS/MS method was found to be suitable to quantify the genotoxic impurity at ppm level present in naproxen drug substance.
\end{abstract}

Keywords: Genotoxic impurity, Alkylating agent, LCMS, Electro spray ionization, Atmospheric chemical ionization.

\section{Introduction}

2-Butyl p-toluenesulfonate is an impurity during the synthesis of naproxen drug substance and the impurity found to be genotoxic alkylating impurity. Alkylating agents has been defined and a detailed discussion made of mechanisms SN1 and SN2 by which they interact with nucleophilic centers ${ }^{1}$. Alkylating agents are used in cancer for the treatment that attaches an alkyl group $\left(\mathrm{C}_{\mathrm{n}} \mathrm{H} 2_{\mathrm{n}+1}\right)$ to DNA ${ }^{2}$. Alkylating agents are used to treat several cancers, however they are toxic to normal cells (cytotoxic) leading to damage bone marrow 
testicals and ovaries and most of the alkylating agents are also carcinogic ${ }^{3-5}$. The most important is alkylation of DNA within the nucleus which leads to cell death. Alkylating agents alkylate within DNA at the N7 position of guanine which is the major cite ${ }^{6}$ resulting in miscoding through abnormal base pairing with thymine leading to strong breakage ${ }^{7}$. Alkylating agents are two types elctrophilic and nucleophilic depends on the character and the two impurities present in naproxen are nucleophilic alkylating agents.

Naproxen is a nonsteroidal anti-inflammatory drugs (NSAIDs). It works by reducing hormones that cause inflammation and pain in the body. Naproxen is used to treat pain or inflammation caused by conditions such as arthritis, ankylosing spondylitis, tendinitis, bursitis, gout or menstrual cramps ${ }^{8}$. Naproxen generally sold as brand names like anaprox , naprelan, naprosyn and aleve. During the synthesis of naproxen drug substance, 2-butyl ptoluenesulfonate used as a raw material which is known to be alkylating agent. This impurity found to be genotoxic/carcinogic ${ }^{3-5}$ hence should be controlled in the naproxen drug substance. The toxicological assessment of these genotoxic impurities and the determination of acceptable limits for such impurities in active substances is a difficult issue and not addressed in sufficient detail in the existing ICH Q3X guidelines ${ }^{9}$. The presence of trace level of the impurity in drug substance or drug product is of genotoxicity concern and has been closely scrutinized by regulatory agencies and pharmaceutical industries ${ }^{10}$.

The 'threshold of toxicological concern' (TTC) of $1.5 \mu \mathrm{g} /$ person/day (exposure of genotoxic impurity in drugs that will be tested or dosed for longer than 12 months) has been suggested by the European Medicines Agency's (EMEA) “Guideline on the limits of genotoxic impurities”11-14 and the PhRMA's white paper ${ }^{15,16}$. Based on the TTC, the concentration limits of genotoxic impurity in drug substances or drug products can then be derived based on the maximum daily dose: concentration limit $(\mathrm{ppm})=[1.5 \mu \mathrm{g} /$ day $)]$ / [dose (g/day)]. For a drug dosed at $1 \mathrm{~g}$ per day, for example, $1.5 \mathrm{ppm}$ would be the limit of a specific genotoxic impurity which would also be the 'target analyte level' (TAL) from an analytical perspective. Given such a low ppm concentration limit, besides the control challenges in process chemistry, developing sensitive and robust methodology for their detection poses a tremendous analytical challenge for the pharmaceutical industry ${ }^{17-19}$.

Therefore it is required for the potential genotoxins to be controlled during the synthesis; where the levels cannot be controlled and no safety data yet exists it may be preferable for the pharmaceutical company to change the route of synthesis of the drug substances $^{20}$. Literature survey reveals that there was no method published for the quantification of 2-butyl p-toluenesulfonate by LC-MS and also it is very difficult to achieve the low level quantification for this impurity using HPLC, GC. By considering the daily intake of naproxen as $1.5 \mathrm{~g}$ /day the regulatory team decided the limit for the impurity is 1 ppm. Quantification at such low level is possible by using triple quad LCMS hence in the present research work a high sensitive LC-MS/MS was developed and described for the quantification of genotoxic impurity at $1 \mathrm{ppm}$ level present in naproxen.

\section{Experimental}

Samples of naproxen, 2-butyl-p-toluenesulfonate (Figure 1) were received from Bulk Actives, Unit-II of Symax Laboratories, Hyderabad, India. HPLC grade Acetonitrile was purchased from J T Baker, Mumbai, India. Ammonium acetate was purchased from Sigma Aldrich, Mumbai, India. High pure water was prepared by using Millipore Milli Q plus purification system (Millipore, USA). 
<smiles>COc1ccc2c(C)c(CC(=O)O)ccc2c1</smiles>

Naproxen (2-(6-methoxy-1-methyl-2-naphthyl) acetic acid)<smiles>CCC(C)OS(=O)(=O)c1ccc(C)cc1</smiles>

Genotoxic impurity (2-butyl p-toluenesulfonate)

Figure 1. Chemical structures and chemical names of naproxen and its genotoxic impurity

\section{Equipment}

The LC-MS method development and validation was carried out using Agilent 1200 series HPLC system connected with Agilent mass spectrometer LC-MS/MS-QqQ system (Agilent technologies, Germany) equipped with Electro spray ionization probe. The data were collected using Agilent mass haunter work station software.

\section{LC-MS chromatographic conditions}

Xterra MS C18 column 50 mm length $\times 4.6 \mathrm{~mm}$ ID with $2.5 \mu \mathrm{m}$ particle size using the isocratic mobile phase of mixture of $10 \mathrm{mM}$ ammonium acetate and acetonirile of 20:80(v/v) at a flow rate of $0.8 \mathrm{~mL} / \mathrm{min}$. Mass spectrometer (MS) was operated in electospray ionization (ESI) positive ion mode with a capillary voltage of $4000 \mathrm{~V}$. The fragmentor was set at $70 \mathrm{~V}$, the drying gas flow was $10 \mathrm{~L} / \mathrm{min}$ with a temperature of $300{ }^{\circ} \mathrm{C}$ and nebuliser pressure was 50 psi. Selected ion mode (SIM) of 173 .The test concentration was about $10 \mathrm{mg} \mathrm{mL}^{-1}$ and the injection volume was $10 \mu \mathrm{L}$. Acetonitrile was used as diluent during the standards and test samples preparations.

\section{Preparation of impurity standard and test sample solution}

The stock solutions of impurity standards are prepared at approximately $1 \mathrm{mg} \mathrm{mL}^{-1}$ in pure diluent. For linearity, the stock solution impurity was diluted using diluent to give standards at $0.4,0.7,1.0,1.2,1.5 \mathrm{ppm}$ with respect to test concentration. The testing naproxen samples were typically prepared at approximately $10 \mathrm{mg} / \mathrm{mL}$ in diluent and sonicated about 10 minutes and filtered through $0.45 \mu$ polytetrafluoroethylene (PTFE) filter.

\section{Results and Discussion}

\section{Optimization of chromatographic conditions}

The main target of LC-MS/MS method was to quantify the genotoxic impurity present in the naproxen drug substance. As volatile buffers required for analysis in LC-MS the mobile phase was restricted to volatile buffers like formic acid, trifluoro acetic acid, ammonium acetate. 
Different trails were made by using these mobile phases and C18 column; the results are showing that ammonium acetate mobile phase shows good sensitivity and separation of genotoxic impurity from naproxen. Various proportions of acetonitrile and ammonium acetate and different concentrations of ammonium acetate tried the impurities shows good sensitivity at $10 \mathrm{mM}$ of ammonium acetate. Using these mobile phases, different columns C8 and C18 trails were made; C18 column shows good peak shape for the impurity. Ammonium acetate is showing most suitable buffer to get more sensitivity for the impurity. The optimized conditions are Xterra MS C18 column $50 \mathrm{~mm}$ length $\times 4.6 \mathrm{~mm}$ ID with 2.5 $\mu \mathrm{m}$ particle size using the isocratic mobile phase of mixture of $10 \mathrm{mM}$ ammonium acetate and acetonirile of 20:80(v/v), diluent is acetonitrile with a run time of 2 minute.

\section{Optimization of MS/MS parameters}

By using the developed LC conditions the impurity solution injected in Electrospray ionization (ESI) and atmospheric pressure chemical ionization (APCI) of triple quad mass spectrometer. The data reveals that, in ESI positive mode the impurity was showing good ionization compared to APCI and the ion is $\mathrm{m} / \mathrm{z} 173[\mathrm{M}+\mathrm{H}]^{+}$in ESI .The ion fragmented using a collision energy, it does not show any stable fragment hence impurity was quantified by Selected ion monitoring (SIM) of 173. Mass parameters optimized are using SIM of 173 are fragmentor voltage $70 \mathrm{~V}$, the drying gas flow was $10 \mathrm{~L} / \mathrm{min}$ with a drying temperature of $300^{\circ} \mathrm{C}$ and nebulizer pressure was 50 psi.

\section{Method Validation}

\section{Linearity}

The linearity of an analytical test procedure is its ability to obtain test results within a given range, which is directly proportional to the concentration of the analyte in the sample ${ }^{21}$. A series of solutions were prepared separately using genotoxic impurity at concentration levels from around detection level to $150 \%$ and the concentration levels are $0.4,0.71 .0,1.2,1.5 \mathrm{ppm}$ respectively with respect to test sample concentration. The peak area versus concentration data was done by linearity plot slop, intercept, and residual sum of squares analysis. The calibration curve was given based on response over the concentration range for the impurity. The correlation coefficient for impurity is 0.992 and the linearity results (Figure 2) are tabulated in Table 1.

Table 1. Results of linearity

\begin{tabular}{ccc}
\hline Concentration, ppm & Area & Mean peak area \\
\hline \multirow{2}{*}{0.4} & 1712 & \\
& 1759 & 1735.5 \\
0.5 & 2188 & \\
& 2101 & 2144.5 \\
0.7 & 2917 & 2966 \\
& 3015 & 4516 \\
1.0 & 4525 & \multirow{2}{*}{6152} \\
& 4507 & \\
Correlation Coefficient & 6119 & 0.992 \\
\hline
\end{tabular}

\section{Limit of Detection (LOD) and Limit of Quantitation (LOQ)}

The quantitation limit of an individual analytical procedure is the lowest amount of analyte in a sample which can be quantitatively determined with suitable precision and accuracy ${ }^{21}$. 
The LOD and LOQ values of impurity were predicted from the linearity data. Each predicted concentration was verified for precision by preparing the solutions at about predicted concentration and injecting each solution six times for LC-MS/MS study and the concentration of LOQ was $0.4 \mathrm{ppm}$ and LOD was 0.12 (Figure 3) and the results are tabulated in Table 2.

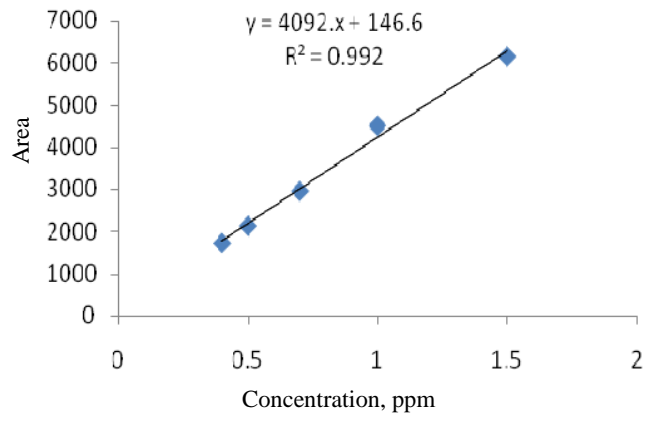

Figure 2. Linearity graph of 2-butyl p-toluenesulfonate
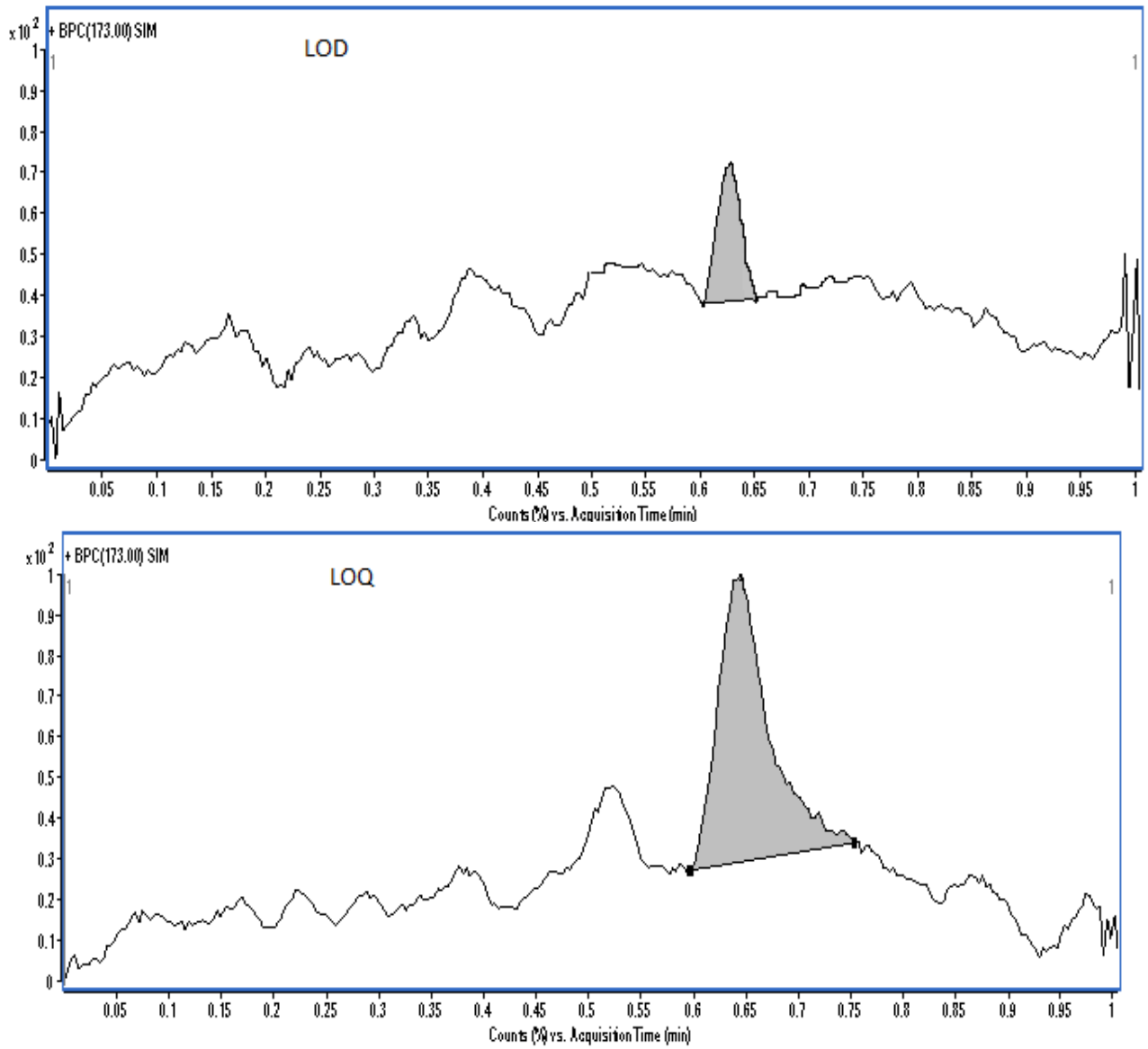

Figure 3. Typical Mass spectrograms of LOD and LOQ of 2-butyl-p-toluenesulfonate 
Table 2. Results of precision

\begin{tabular}{cccc}
\hline \multirow{2}{*}{ Preparation } & \multicolumn{3}{c}{ Impurity } \\
\cline { 2 - 4 } & Area (LOQ) & Area (100\%) & Area (150\%) \\
\hline 1 & 1798 & 4819 & 7142 \\
2 & 1825 & 4728 & 7233 \\
3 & 1755 & 4999 & 7177 \\
4 & 1819 & 4785 & 7028 \\
5 & 1922 & 4877 & 7219 \\
6 & 1879 & 4825 & 7222 \\
Average & 1833.0 & 4838.8 & 7099 \\
SD & 59.3 & 92.6 & 77.6 \\
\%RSD & 3.2 & 1.9 & 1.1 \\
\hline
\end{tabular}

\section{Precision}

The precision of an analytical procedure expresses the closeness of agreement between a series of measurements from multiple sampling of the homogeneous sample under prescribed conditions ${ }^{21}$. The precision of the method was checked by preparing test solutions by spiking the impurities at LOQ, 50\%, 75\%, 100\%, 125\% and 150\% level with the drug substance for six times and injected each once also injected $100 \%$ spiked solution for 6 times to show the system precision. The \% RSD of the areas is within $1.1 \%$ and $3.2 \%$ confirming the good precision of the developed method.

\section{Accuracy}

The accuracy of an analytical procedure expresses the closeness of agreement between the value which is accepted either as a conventional true value or an accepted reference value and the expected value found ${ }^{21}$. The accuracy of the method was evaluated in sample solutions were prepared in triplicate by spiking both the impurities at LOQ level, 50\%, 75\%, 100\%, 125\% and $150 \%$ with naproxen drug substance and injected each solution in to LC-MS as per methodology. The percentage of recovery was calculated and the values are within $94.5 \%$ to $96.1 \%$ and at ppm levels these recoveries were satisfactory and the results are tabulated in Table 3.

Table 3. Results of accuracy study

\begin{tabular}{ccccccc}
\hline Level & Amount Added, $\mu$ g Amount found, $\mu g$ & \% Recovery & Mean & SD & \%RSD \\
\hline LOQ Sample-1 & \multirow{2}{*}{0.406} & 0.388 & 95.6 & & & \\
LOQ Sample-2 & 0.375 & 92.4 & 94.7 & 2.1 & 2.2 \\
LOQ Sample-3 & & 0.391 & 96.3 & & & \\
50\% Sample-1 & \multirow{2}{*}{0.508} & 0.511 & 100.6 & & & \\
50\% Sample-2 & 0.485 & 95.5 & 96.5 & 3.7 & 3.8 \\
50\% Sample-3 & & 0.475 & 93.5 & & & \\
75\% Sample-1 & \multirow{2}{*}{0.762} & 0.715 & 93.8 & & & \\
75\% Sample-2 & 0.732 & 96.1 & 95.6 & 1.6 & 1.6 \\
75\% Sample-3 & & 0.738 & 96.9 & & & \\
100\% Sample-1 & \multirow{2}{*}{1.016} & 0.975 & 96.0 & & & \\
100\% Sample-2 & 0.997 & 98.1 & 96.1 & 1.9 & 2.0 \\
100\% Sample-3 & & 0.958 & 94.3 & & & \\
150\% Sample-1 & \multirow{2}{*}{1.523} & 1.452 & 95.3 & & & \\
150\% Sample-2 & 1.489 & 97.8 & 96.6 & 1.2 & 1.3 \\
150\% Sample-3 & & 1.472 & 96.7 & & & \\
\hline
\end{tabular}




\section{Robustness}

To evaluate the robustness of the developed LC-MS method, the LC conditions like flow rate, ratio of the composition of the mobile phase, and mass parameters like gas flow, drying gas temperature, collision energy were slightly altered and injected $100 \%$ impurity spiked solution of naproxen. In all the varied condition of LC and MS the method shows required sensitivity to quantify the impurity. The method was repeated in different days with different columns, analysts and tested the number of quality control batches of Naproxen samples and the results revealed that robustness of the method (Table 4).

Table 4. Results of Robustness study

\begin{tabular}{|c|c|c|c|c|}
\hline S.No & Parameter & Variation & $\begin{array}{c}\text { \%RSD of area of } \\
\text { impurity peak }(0.4 \mathrm{ppm})\end{array}$ & $\begin{array}{c}\text { \%RSD of Area of } \\
\text { impurity peak(1 ppm) }\end{array}$ \\
\hline \multirow[b]{2}{*}{1} & \multirow{2}{*}{$\begin{array}{l}\text { Column temperature } \\
\left( \pm 5^{\circ} \mathrm{C} \text { of set }\right. \\
\text { temperature })\end{array}$} & a) At $35^{\circ} \mathrm{C}$ & 2.5 & 1.9 \\
\hline & & b) At $45^{\circ} \mathrm{C}$ & 2.2 & 2.1 \\
\hline \multirow[t]{2}{*}{2} & \multirow{2}{*}{$\begin{array}{c}\text { Flow rate } \\
( \pm 0.1 \mathrm{~mL} \text { of set flow })\end{array}$} & $\begin{array}{l}\text { a) At } 0.9 \\
\mathrm{~mL} / \mathrm{min}\end{array}$ & 3.1 & 2.0 \\
\hline & & $\begin{array}{l}\text { b) At } 1.1 \\
\mathrm{~mL} / \mathrm{min}\end{array}$ & 2.7 & 1.2 \\
\hline \multirow{2}{*}{3} & Nebuliser pressure & a) At $45 \mathrm{psi}$ & 2.1 & 2.2 \\
\hline & ( \pm 5 psi of set value) & b) At 55 psi & 2.5 & 1.5 \\
\hline \multirow{2}{*}{3} & Drying gas temerature & a) At $295^{\circ} \mathrm{C}$ & 2.2 & 1.8 \\
\hline & ( $\pm 5^{\circ} \mathrm{C}$ of set value) & b) At $305^{\circ} \mathrm{C}$ & 2.8 & 2.2 \\
\hline \multirow{2}{*}{3} & \multirow{2}{*}{$\begin{array}{l}\text { Drying gas flow } \\
\text { ( } \pm 1 \text { L of set value) }\end{array}$} & $\begin{array}{l}\text { a) At } 9 \\
\text { L/min }\end{array}$ & 1.8 & 2.1 \\
\hline & & $\begin{array}{l}\text { a) At } 11 \\
\mathrm{~L} / \mathrm{min}\end{array}$ & 0.9 & 1.8 \\
\hline
\end{tabular}

\section{Conclusion}

In this paper a sensitive specific, accurate, validated and well-defined LC-MS/MS method for the quantification of suspected genotoxic alkylating impurity 2-butyl $p$-toluenesulfonate at $1 \mathrm{ppm}$ level in naproxen drug substance was described and the limit of quantification found to be $0.4 \mathrm{ppm}$. The developed method is highly specific and sensitive reliable technique for the quantification of the gentoxic impurity2-butyl-p-toluenesulfonate in the naproxen drug substance. The method is well suited for the quantitation of the genotoxic impurity present in naproxen during quality control testing.

\section{Acknowledgment}

The authors wish to thank the Professors and Research Scholars for supporting this work. We would also like to thank colleagues in separation science division of Analytical Research Matrix Laboratories Limited for their cooperation in carrying out this work.

\section{References}

1. Warwick G P, Cancer Res., 1963, 23, 1315-1333.

2. http://faculty.swosu.edu/scott.long/phcl/antineop.htm; Retrieved on 2009-01-24.

3. Wiedemann G J, Robins H I, Gutsche S, Mentzel M, Deeken M, Katschinski D M et al., Eur J Cancer, 1996, 32A(5), 888-892. 
4. Doll $\mathrm{R}$ and Peto R J, J Natl Cancer Inst., 1981, 66(6), 1192-1308; DOI:10.1093/jnci/66.6.1192

5. Bartsch H. and Montesano R, Carcinogenesis, 1984, 5(11), 1381-1393; DOI:10.1093/carcin/5.11.1381

6. Charles C. Price, Gaucher G M, Koneru P, Shibakawa R, Sowa J R, Yamaguchi M, Article first published online: 16 DEC 2006; DOI: 10.1111/j.17496632.1969.tb24877.x

7. Kondo N, Takahashi A, Ono K and Ohnishi T, J Nucleic Acids, 2010; DOI: 10.4061/2010/543531

8. $\quad$ Todd P A and Clissold S P, Drugs, 1990, 40(1), 91-137.

9. ICH, Q3A (R2) Guideline on Impurities in New Drug Substances, 2006. IFPMA, Geneva

10. Lutz Muller, Robert J Mauthe, Christopher M Riley, Marta M Andino, David De Antonisd and Chris Beels et al., Regulatory Toxicology Pharm., 2006, 44, 198-211.

11. Guidelines on the Limits of Genotoxic Impurities. Committee for Medicinal Products or Human Use [CHMP]. European Medicines Agency [EMEA] 2006:28. (CPMP/SWP/5199/02, EMEA/CHMP/QWP/ 251344/2006).

12. Kroes R, Renwick A G, Cheeseman M, Kleiner J, Mangelsdorf I, Piersma A, Schilter B, Schlatter J, van Schothorst F, Vos J G and Würtzen G, Food Chem Toxicol., 2004, 42(1), 65-83; DOI:10.1016/j.fct.2003.08.006

13. McGovern T and Jacobson- Kram D, TrAC Trends Anal Chem., 2006, 25(8), 790-795; DOI:10.1016/j.trac.2006.06.004

14. European Medicines Evaluation Agency, Committee for Medicinal Products for Human Use (CHMP), Guideline on the limits of genotoxic impurities, 2004, CPMP/SWP/5199/02, London, UK.

15. ICH M7, Assessment and Control of DNA Reactive (Mutagenic) Impurities in Pharmaceuticals to Limit Potential Carcinogenic Risk, Step 2 draft guideline, February 2013.

16. Delaney E J, Reg Toxicol Pharm., 2007, 49(2), 107-124;

DOI:10.1016/j.yrtph.2007.06.008

17. Dobo K L, Greene N, Cyr M O, Caron S and Ku W W, Reg Toxicol Pharma., 2006, 44(3), 282-293; DOI:10.1016/j.yrtph.2006.01.004

18. Colon I and Richoll S M, J Pharm Biomed Anal., 2005, 39(3-4), 477-485; DOI:10.1016/j.jpba.2005.04.037

19. Li H and Sluggett G W, J Pharm Biomed Anal., 2005, 39(3-4), 486-494; DOI:10.1016/j.jpba.2005.04.042

20. Elder D P, Lipczynski A M and Teasdale A, J Pharm Biomed Anal., 2008, 48(3), 497-507; DOI:10.1016/j.jpba.2008.06.009

21. International Conference on Harmonization (ICH) guideline, Text on validation of analytical procedures, Q2A (R1), 1996. IFPMA, Geneva. 